Sādhanā Vol. 36, Part 4, August 2011, pp. 525-550. (C) Indian Academy of Sciences

\title{
Balanced gait generations of a two-legged robot on sloping surface
}

\author{
PANDU RANGA VUNDAVILLI and DILIP KUMAR PRATIHAR* \\ Department of Mechanical Engineering, Indian Institute of Technology, \\ Kharagpur 721 302, India \\ e-mail: pandu@mech.iitkgp.ernet.in; dkpra@mech.iitkgp.ernet.in
}

MS received 2 February 2010; revised 14 December 2010; accepted 25 Feburary 2011

\begin{abstract}
In this paper, dynamically balanced gait generation problem of a 7-DOF two-legged robot moving up and down through the sloping surface is presented. The gait of the lower links during locomotion is obtained after assuming suitable trajectories for the swing leg and hip joint. The trunk motion is initially generated based on the concept of static balance, which is different from the well-known semi-inverse method and then checked for its dynamic balance calculated using the concept of Zero-Moment Point (ZMP). Lagrange-Euler formulation is attempted for the determination of joint torques. Average power consumption at each joint is then determined based on the computed torques. Moreover, the variations of dynamic balance margin and average power consumption are studied for both ascending and descending through the sloping surface. Both of them are found to be more for the ascending gait generation compared to those for the descending case. The effects of variations of the slope have also been studied on the average dynamic balance margin and power consumption for both the cases.
\end{abstract}

Keywords. Biped robot; sloping surface; dynamic balance; power consumption.

\section{Introduction}

Biped robots and their locomotion have received much attention, nowadays, as they are expected to be used in the environment designed for the human beings to help them in the day-to-day life. Many gait generation and control algorithms have been developed based on the assumption that the walking surface is perfectly flat (Vukobratovic et al 1970; Juricic \& Vukobratovic 1972; Furusho \& Masubuchi 1987; Takanishi et al 1989; Seo \& Yoon 1995; Silva \& Tenreiro Machado 1998; Goswami 1999; Lum et al 1999; Sugihara et al 2002; Lim et al 2002; Pleaten et al 2003; Kajita et al 2003; Vukobratovic \& Radic 2004; Kim et al 2005). In reality, however, a typical environment may also contain some sloping surfaces and staircases. During

*For correspondence 
locomotion, the biped robot should be dynamically balanced, during which the Zero-Moment Point (ZMP) should not fall outside the support foot in case of single support phase (considered in the present study). However, in case of double support phase (not studied in the present paper), the biped robot might be dynamically balanced, even if the ZMP does not fall inside any one of two support feet. The dynamic balance is measured using a parameter called Dynamic Balance Margin (DBM), which is calculated as the shortest distance between the edge of the support foot and the ZMP.

Many researchers contributed towards the gait generation and walking control of a biped robot on flat surface. Some of these attempts are discussed below. Semi-inverse method was proposed by Juricic \& Vukobratovic for determining the trunk motion, in which the position of ZMP was defined first. Furusho \& Masubuchi (1987) developed a new reduced order model of a biped locomotion system based on the concept of local feedback. Takanishi et al (1989) introduced a control method for dynamic biped walking and it was tested on WL-12R robot. Seo \& Yoon (1995) proposed a technique based on the concept of DBM for designing a robust dynamic gait. Silva \& Tenreiro Machado (1998) presented the energy analysis of a bipedal walking system and studied the effect of locomotion variables on the energy flow. Goswami (1999) proposed the concept of Foot Rotation Indicator (FRI) point, which is an indication of postural imbalance and it should be avoided. Lum et al (1999) developed computed torque control scheme and variable structure control law for the control of a biped robot and evaluated the schemes on a plane surface and climbing up a flight of stairs. Sugihara et al (2002) generated real-time humanoid motion, in which the Center of Gravity (CG) was controlled by indirect manipulation of the ZMP. On-line walking pattern for a biped robot with trunk was developed by Lim et al (2002), in which the lower-links motion pattern was generated first and then the trunk and waist motions were realized by a stabilization control based on ZMP trajectory. Pleaten et al (2003) developed asymptotically balanced walking based on Poincare's method and analysed the existence and balance of periodic orbits introduced by the controller. Kajita et al (2003) modelled the dynamics of the biped robot as the running cart on a table and formulated as a ZMP tracking problem to achieve dynamic walking patterns. Vukobratovic \& Radic (2004) proposed an integrated dynamic control scheme of humanoid robots based on spatial dynamic model of humanoid mechanism, model of servosystem and environment model. The structure of dynamic controller involved four feedback loops, namely position-velocity feedback of the robotic mechanism joints, dynamic reaction feedback around ZMP, contact force feedback at the moment of the foot striking the ground and load feedback at the mechanism joints. Kim et al (2005) developed the KHR-2 humanoid robot and achieved dynamic walking. Distributed control architecture was used to control all the joint axes effectively.

In all the above studies, biped walking was considered on a flat surface. The gait generation and walking control problem on the inclined and uneven surfaces were also solved by some of the researchers. Zheng et al (1998) proposed a scheme for the biped robot to climb the sloping surfaces using position sensors on the joints and force sensors underneath the heel and toe for static walking, quasi-dynamic and dynamic walking (Zheng \& Shen 1990). Kajita \& Tani (1991) used the concept of linear inverted pendulum mode for the dynamic biped locomotion on rugged terrain. Boone \& Hodgins (1997) proposed slipping and tripping reflexes for bipedal robots for the single-slip tasks with varying static friction coefficients and single-trip tasks with varying obstacle heights. Shih et al studied the motion control of a statically stable biped robot on an uneven floor by assuming that the characteristics of the terrain were already known in advance. Ono et al (1998) developed an algorithm to realize a stable walking motion on an unknown slope. The slope angle was detected with the help of touch sensors mounted on the soles and encoders fixed on the joint. 
Pratt et al (2001) proposed a virtual model control for the biped robot walking on both the level as well as sloping terrain based on geometric considerations, in which the ground was detected using foot contact switches. Zhou et al (2004) formulated the gait synthesis problem of biped locomotion as a constrained optimization problem, considering ZMP for dynamically balanced locomotion, internal forces for smooth transition and geometric constraints for walking. Sugahara et al (2005) proposed a walking control method consisting of a position control, virtual compliance control and posture control for the biped locomotor on an inclined plane. Kim et al (2007) also developed walking control algorithm for biped humanoid robot on uneven and inclined surface after considering local and global inclinations of the floor. Suitable walking pattern was designed first and on-line controllers were then developed to activate the walking patterns at suitable periods.

The staircase ascending and descending gait generation problem of a biped robot was solved by Vundavilli et al (2007), after utilizing the concept of static balance for the trunk motion generation and ZMP for verifying the dynamic balance. The difference between the said previous work on moving through the staircase and the present work lies in the following facts:

- In case of moving through the staircase, the feet are placed on the flat surface, whereas they are put on the inclined plane while walking along a sloping surface.

- The static friction coefficient between the foot and sloping surface and the angle of slope play some vital roles to ensure the movement without slipping on an inclined plane.

- The projected area of foot support polygon reduces with the angle of slope and it has significant influence on the DBM.

In the present work, dynamically balanced gaits of a biped robot ascending and descending the sloping surface have been developed. In the proposed method, an algorithm is used to generate the motion of the lower links, after assuming suitable trajectories for the swing leg and hip joint. The concept of static balance is utilized to generate the motion of the trunk for the first half of the cycle and is then verified for its dynamic balance. Once the dynamic balance is maintained for the first half of the cycle, the trunk motion for the next half of the cycle is generated following some repeatability conditions. The generated ascending and descending gaits are compared with respect to the obtained dynamic balance margin and average power consumption. Moreover, the effect of slope on dynamic balance margin and average power consumption is studied in detail, for both the ascending and descending gait generations of the biped robot moving through the sloping surface.

The rest of the manuscript is organized as follows: Section 2 explains the mathematical formulation of the problem. Results are presented in section 3. Some concluding remarks are made in section 4 and the scope for future work is discussed in section 5.

\section{Mathematical formulation}

The present paper deals with the dynamically balanced gait generation problem of a two-legged robot, while moving up and down the sloping surface. Figure 1 shows a 7 degrees of freedom (DOF) (two at the ankles, two at the knee and three at the hip) biped robot considered in this study along with the lumped masses attached to the corresponding links. All the joints are considered to be rotary in nature. The balance of the robot is checked only along the direction of movement that is, in the forward direction. This paper deals with two cases-Case 1: ascending the sloping surface and Case 2: descending the sloping surface, which are explained below. 


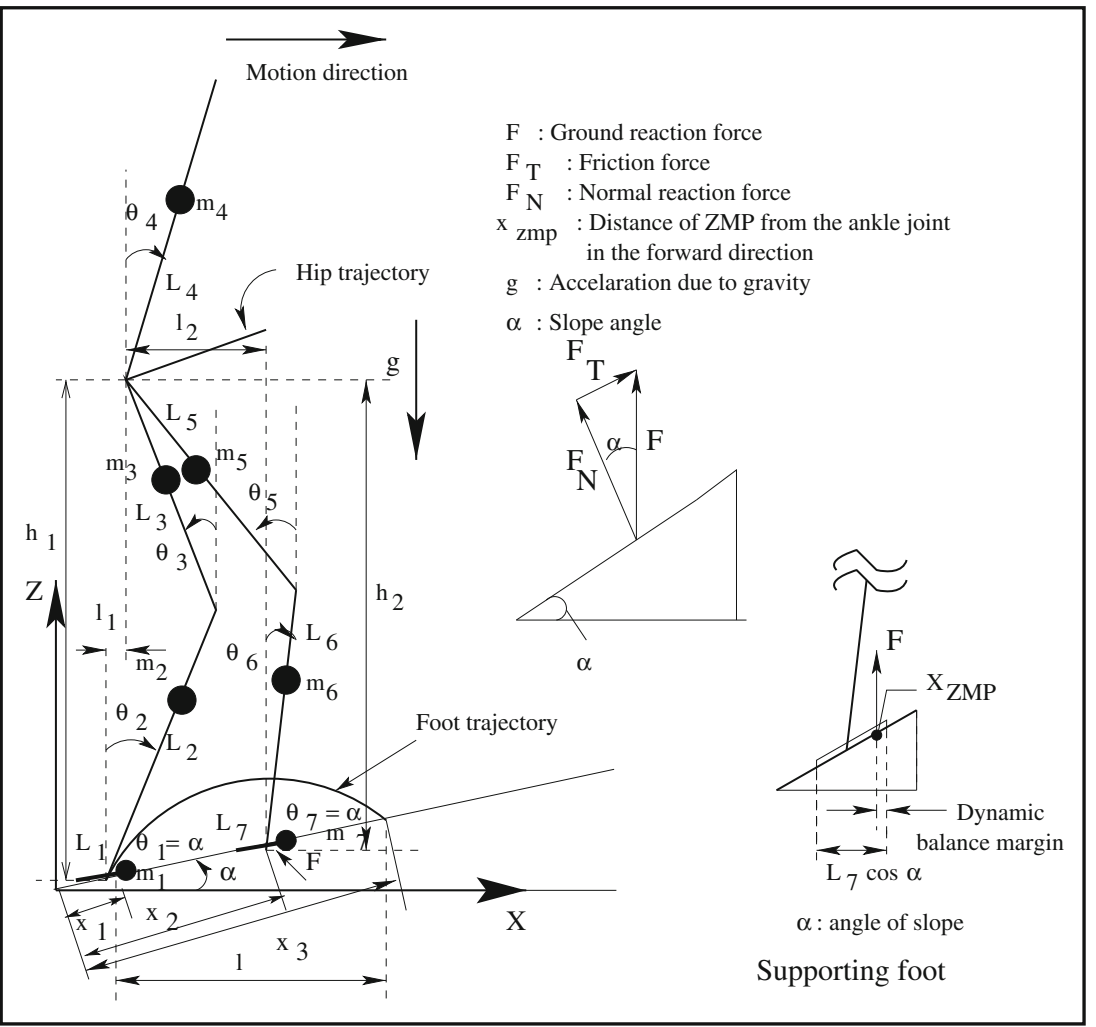

Figure 1. A schematic view of a two-legged robot(7-DOF) moving up the sloping surface.

\subsection{Ascending the sloping surface}

Two important parameters: step length $l$ and hip height $h$ can be determined for the known feet placements $\left(x_{1}, x_{2}, x_{3}\right)$, slope of the surface $\alpha$, length of the links $L_{2}$ and $L_{3}$, joint angles of the swing leg $\left(\theta_{2}, \theta_{3}\right)$ as follows:

$$
\begin{gathered}
l=x_{3} \cos \alpha-x_{1} \cos \alpha, \\
h_{1}=L_{2} \cos \theta_{2}+L_{3} \cos \theta_{3} .
\end{gathered}
$$

2.1a Gait generation: The swing foot is assumed to follow a cubic polynomial trajectory, so that it can reach safely to the next landing position after avoiding collision with the sloping surface. The equation of the swing foot trajectory (that is, the trajectory of ankle joint) has been considered to be as follows:

$$
z=c_{0}+c_{1} x+c_{2} x^{2}+c_{3} x^{3}
$$

where $z$ is the height of swing foot at a distance $\mathrm{x}$ from the starting point and $c_{0}, c_{1}, c_{2}$ and $c_{3}$ are the coefficients, whose values are determined with the help of the following boundary conditions:

- at $x=x_{1} \cos \alpha, z=x_{1} \sin \alpha$, 
- at $x=\left(x_{1} \cos \alpha+x_{2} \cos \alpha\right) / 2, z=x_{2} \sin \alpha+\frac{f_{s}}{2}$,

- at $x=\left(x_{2} \cos \alpha+x_{3} \cos \alpha\right) / 2, z=x_{3} \sin \alpha+\frac{f_{s}}{2}$,

- at $x=x_{3} \cos \alpha, z=x_{3} \sin \alpha$,

where $\alpha$ and $f_{s}$ are the angle of slope and length of the foot, respectively. Appendix A shows the values of the coefficients $c_{0}, c_{1}, c_{2}$ and $c_{3}$ obtained using the above boundary conditions. Figure 2 shows the variation of velocity of swing leg with time. The duration of maximum value of each time step $(T)$ is assumed to be equal to $5.0 \mathrm{~s}$. The maximum velocity $\left(V_{\max }\right)$ attained by the swing leg in that cycle is set equal to $0.056 \mathrm{~m} / \mathrm{s}$. The maximum velocity and time step have been decided based on the assumed capacity of the motors. In each time step, the swing leg is allowed to move with acceleration for the first one second, with constant velocity for the next three seconds and deceleration for the last one second. The trajectory of the hip joint is assumed to follow a straight line with the slope kept equal to that of the surface, in order to generate the repeatable gait for the next motion cycle. Hip joint as well as swing foot trajectories are divided into seven equal time intervals for the purpose of study. The angles $\theta_{2}$ and $\theta_{3}$ can be calculated using the mathematical expressions given below:

$$
\theta_{2}=\sin ^{-1}\left(\frac{h_{1} L_{3} \sin \psi_{1}+l_{1}\left(L_{2}+L_{3} \cos \psi_{1}\right)}{\left(L_{2}+L_{3} \cos \psi_{1}\right)^{2}+\left(L_{3} \sin \psi_{1}\right)^{2}}\right),
$$

where $h_{1}$ and $l_{1}$ are shown in figure $1 ; L_{2}$ and $L_{3}$ are the lengths of two links; and $\psi_{1}$ has been calculated using the expression: $\psi_{1}=\arccos \left(\left(h_{1}^{2}+l_{1}^{2}-L_{2}^{2}-L_{3}^{2}\right) / 2 L_{2} L_{3}\right)$.

Thus, $\theta_{3}$ can be calculated from the equation $\theta_{3}=\theta_{2}-\psi_{1}$. Similarly, the angles $\theta_{5}$ and $\theta_{6}$ are calculated using the hip height $h_{2}$ and distance of the supporting ankle from the projection of hip joint (that is, $l_{2}$ ) as shown in figure 1 . The mathematical expressions used for calculating the joint angles $\theta_{5}$ and $\theta_{6}$ are shown below:

$$
\theta_{6}=\sin ^{-1}\left(\frac{h_{2} L_{5} \sin \psi_{2}+l_{2}\left(L_{6}+L_{5} \cos \psi_{2}\right)}{\left(L_{6}+L_{5} \cos \psi_{2}\right)^{2}+\left(L_{5} \sin \psi_{2}\right)^{2}}\right),
$$

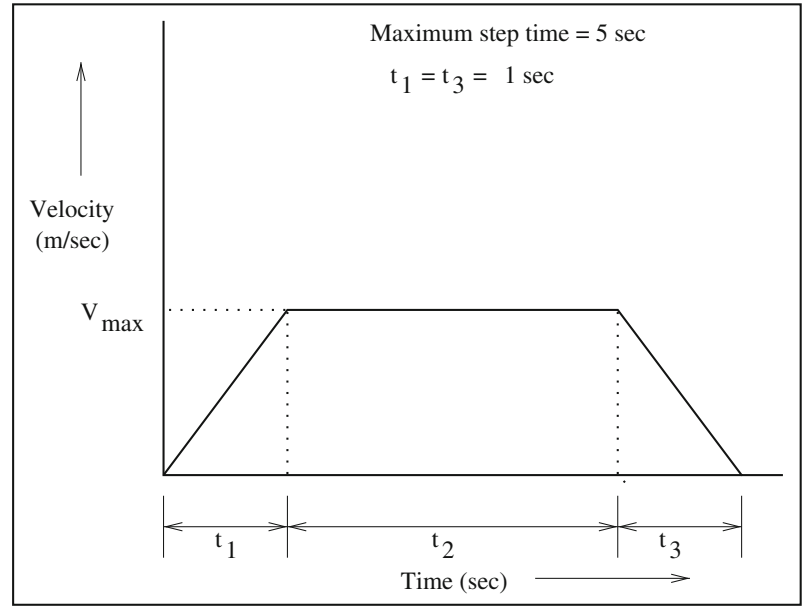

Figure 2. Velocity distribution of the foot of the swing leg in one time step. 
where $L_{5}$ and $L_{6}$ are the lengths of the links (refer to figure 1); $\psi_{2}=\arccos \left(\left(h_{2}^{2}+l_{2}^{2}-L_{6}^{2}-\right.\right.$ $\left.L_{5}^{2}\right) / 2 L_{6} L_{5}$ ). The angle $\theta_{5}$ can be calculated using the expression $\theta_{5}=\theta_{6}-\psi_{2}$. To ensure a cyclic gait, the following repeatability conditions are to be followed by the lower links:

$$
\begin{aligned}
& \theta_{2, \text { initial }}=\theta_{6}, \text { final }, \\
& \dot{\theta}_{2, \text { initial }}=\dot{\theta}_{6}, \text { final }, \\
& \theta_{3, \text { initial }}=\theta_{5}, \text { final }, \\
& \dot{\theta}_{3, \text { initial }}=\dot{\theta}_{5, \text { final }} .
\end{aligned}
$$

The trunk motion is generated using the concept of static balance (Vundavilli et al 2007), which is different from the most popular semi-inverse method (Juricic \& Vukobratovic 1972; Vukobratovic et al 1990) and verified for its dynamic balance after determining the position of ZMP. The mathematical expression for trunk motion, that is, $\theta_{4}$ obtained from the concept of static balance is as follows:

$$
\theta_{4}=\sin ^{-1}\left(\frac{1}{m_{4} r_{4}}\left(\left(p \times m_{\text {sum }}\right)-\left(m_{1} d_{1}+m_{2} d_{2}+m_{3} d_{3}+m_{5} d_{5}+m_{6} d_{6}+m_{7} d_{7}\right)\right)\right),
$$

where $p$ denotes the distance of projected mass centers from the ankle joint and $d_{1}, d_{2}, d_{3}, d_{5}$, $d_{6}$ and $d_{7}$ represent the distances of masses $m_{1}, m_{2}, m_{3}, m_{5}, m_{6}$ and $m_{7}$, respectively, from the projection of respective mass center, $r_{4}$ indicates the distance of $m_{4}$ from the hip joint and $m_{\text {sum }}=m_{1}+m_{2}+m_{3}+m_{4}+m_{5}+m_{6}+m_{7}$. It is important to mention that the value of $p$ (refer to equation (6)) should not exceed the half of support foot length to maintain the static balance of the robot. The trunk motion is generated for the first half of the cycle and it will be verified for its dynamic balance. If the system is found to be dynamically balanced for the first half of the cycle, the trunk motion for the next half of the cycle is generated based on the repeatability conditions as given below:

$$
\begin{aligned}
& \theta_{4, \text { initial }}=\theta_{4, \text { final },} \\
& \dot{\theta}_{4, \text { initial }}=\dot{\theta}_{4, \text { final }} .
\end{aligned}
$$

To summarize, the following steps have been used to generate the trunk motion:

Step 1: Determine the trunk motion of the robot after considering the static balance for the first half of the cycle.

Step 2: Verify, whether the entire system is dynamically balanced for the above trunk motion at different intervals of the half cycle by determining the position of ZMP, which has been discussed in the next sub-section.

Step 3: If the system is found to be dynamically balanced for the first half of the cycle, the trunk motion for the next half of the cycle is obtained using the repeatability conditions and terminate the program. Otherwise, go to step 4.

Step 4: If the system is not found to be dynamically balanced, modify the motion of the trunk besides that of the ankle, knee and hip joints, and repeat steps 1 through 3 .

2.1b Dynamic balance analysis: In the present work, dynamic balance of the robot on the sloping surface has been decided based on two different criteria. The first one is based on static friction coefficient $\mu$ and the second one uses the position of the ZMP. For a particular value of 
static friction of coefficient, the maximum angle of slope along which the biped robot can walk without slipping has been determined. The graphical relationship among tangential force $F_{T}$, normal force $F_{N}$, and angle of slope $\alpha$ is shown in figure 1. The mathematical expressions for $F_{T}$ and $F_{N}$ are given as follows:

$$
\begin{gathered}
F_{T}=F \sin (\alpha), \\
F_{N}=F \cos (\alpha),
\end{gathered}
$$

where $F$ represents the ground reaction force and $\alpha$ is the angle of slope. The maximum value of slope angle along which the robot can walk without slipping is decided by the static friction coefficient $\mu$. If the angle of slope $\alpha$ becomes greater than $\phi$ (where $\phi=\arctan (\mu)$ ), the biped robot will start slipping along the sloping surface. For the dynamically balanced gait, the ZMP should lie inside the projected sole of the supporting foot. The position of the ZMP from the ankle joint of the ground leg in the direction of motion $\left(x_{Z M P}\right)$ can be determined as follows:

$$
x_{Z M P}=\frac{\sum_{i=1}^{7}\left(I_{i} \dot{\omega}_{i}+m_{i} x_{i}\left(\ddot{z}_{i}-g\right)-m_{i} \ddot{x}_{i} z_{i}\right)}{\sum_{i=1}^{7} m_{i}\left(\ddot{z}_{i}-g\right)},
$$

where $I_{i}$ represents the moment of inertia of $i^{t h} \operatorname{link}\left(\mathrm{kg}-\mathrm{m}^{2}\right), \dot{\omega}_{i}$ denotes the angular acceleration of link $i\left(\mathrm{rad} / \mathrm{s}^{2}\right), m_{i}$ denotes the mass of $i$-th link $(\mathrm{kg}),\left(x_{i}, y_{i}, z_{i}\right)$ is the coordinate of $i^{\text {th }}$ lumped mass, $\mathrm{g}$ indicates the acceleration due to gravity $\left(\mathrm{m} / \mathrm{s}^{2}\right), \ddot{z}_{i}$ is the acceleration of link $i$ in $\mathrm{z}$-direction $\left(\mathrm{m} / \mathrm{s}^{2}\right), \ddot{x}_{i}$ is the acceleration of link $i$ in X-direction $\left(\mathrm{m} / \mathrm{s}^{2}\right)$.

The ZMP should not lie outside the foot support polygon (in case of single support phase) to maintain the dynamic balance of the robot, otherwise the ankle, knee and hip joint torques of the supporting leg must be updated to move the ZMP to a safe zone. The Dynamic Balance Margin (DBM) is defined as the shortest distance between the end of the supporting polygon and the point where the ZMP is acting. The expression utilized for determining the DBM in the direction of motion $\left(x_{D B M}\right)$ is given below.

$$
x_{D B M}=\left(\frac{L_{7}}{2} \cos \alpha-\left|x_{Z M P}\right|\right),
$$

where $L_{7}$ is the length of the supporting foot.

2.1c Torques and average power consumption: Lagrange-Euler formulation is used to solve the dynamics of the biped robot. Figure 3 shows the D-H parameters setting ( $\mathrm{Fu}$ et al 1987) at different joints of the robot. The joint angles are considered as positive, when measured in the clockwise sense with respect to the vertical axis. In D-H parameters setting, the ankle angle of the swing leg is considered as positive, when it is measured in the anti-clockwise sense with respect to horizontal axis. Then, the relationship between the $\mathrm{D}-\mathrm{H}$ parameters angles and the gait angles are as follows. $q_{1}=\theta_{1}=\alpha$ (as there is no variation), $q_{2}=\left(90-\theta_{2}-\theta_{1}\right), q_{3}=$ $\left(\theta_{2}-\theta_{3}\right), q_{4}=\left(\theta_{3}-\theta_{4}\right), q_{5}=\left(\theta_{4}-\theta_{5}\right), q_{6}=\left(\theta_{5}-\theta_{6}\right), q_{7}=\left(\theta_{7}-\left(90-\theta_{6}\right)\right)$. The joint angles are assumed to follow fifth order polynomial for smooth variation, as given below:

$$
q_{i}(t)=a_{i 0}+a_{i 1} t+a_{i 2} t^{2}+a_{i 3} t^{3}+a_{i 4} t^{4}+a_{i 5} t^{5},
$$

where $a_{i 0}, a_{i 1}, a_{i 2}, a_{i 3}, a_{i 4}$ and $a_{i 5}$ are the coefficients. It is to be noted that the cycle time has been equally divided into seven intervals using eight points. As there are six coefficients in 


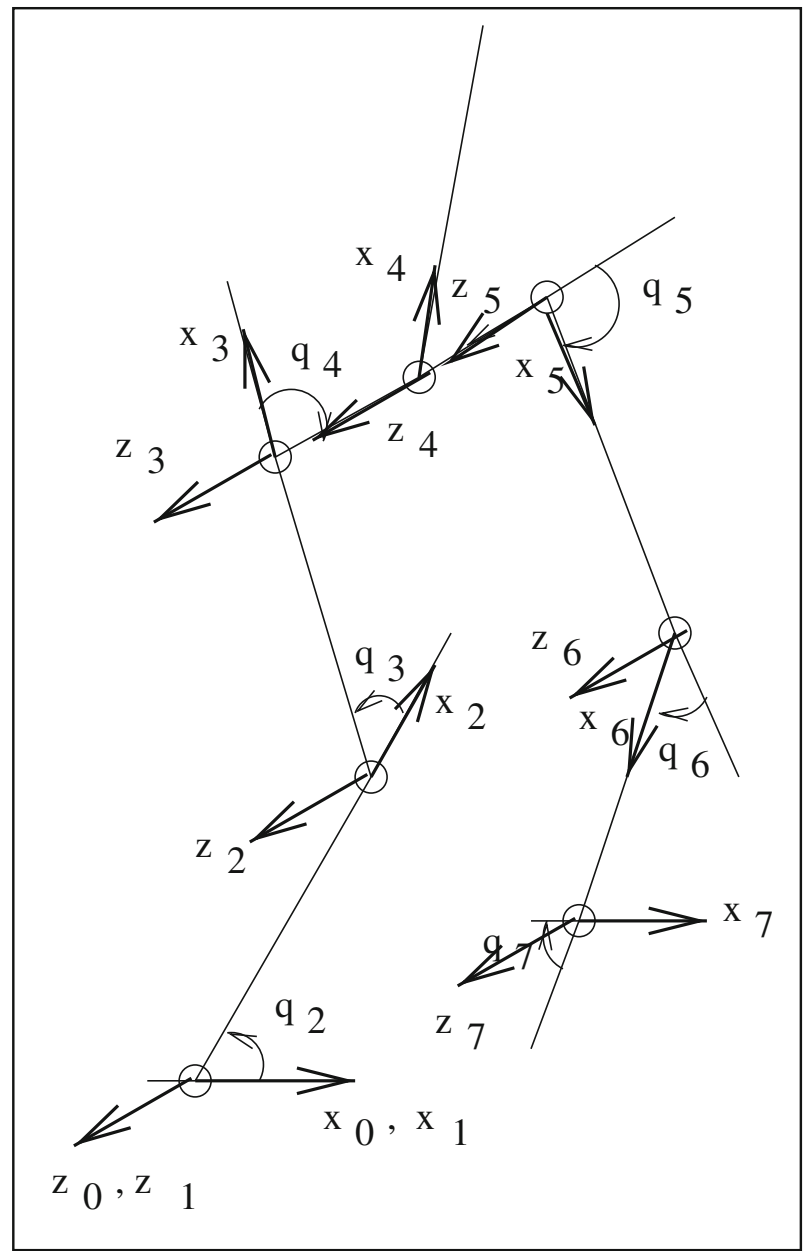

Figure 3. A schematic diagram showing D-H parameter setting and included angles between the links.

equation (9), $q_{i}$ values at six instants only (that is, initial, final and 2 nd, 4th, 5th and 7 th instants) are considered to determine the values of coefficients of the above equation and $i=1,2, \cdots, n$ joints. The above equation has been used to determine the variations of angular velocity and acceleration in a cycle. The joint torques $(\tau)$ can be determined as follows:

$$
\begin{gathered}
\tau_{i}=\sum_{k=1}^{n} D_{i k} \ddot{\mathbf{q}}_{k}+\sum_{k=1}^{n} \sum_{m=1}^{n} h_{i k m} \dot{\mathbf{q}}_{k} \dot{\mathbf{q}}_{m}+C_{i}, \quad i=1,2, \cdots, n, \\
\text { where } \mathrm{n}=\text { number of joints, } \\
D_{i k}=\sum_{j=\max (i, k)}^{n} \operatorname{Tr}\left(\mathbf{U}_{j k} \mathbf{J}_{j} \mathbf{U}_{j i}^{T}\right) \quad i, k=1,2, \cdots, n,
\end{gathered}
$$




$$
\begin{gathered}
h_{i k m}=\sum_{j=\max (i, k, m)}^{n} \operatorname{Tr}\left(\mathbf{U}_{j k m} \mathbf{J}_{j} \mathbf{U}_{j i}^{T}\right) \\
C_{i}=\sum_{j=1}^{n}\left(-m_{j} \mathbf{g} \mathbf{U}_{j i} \overline{\mathbf{r}}_{j}^{j}\right) \quad i, k, m=1,2, \cdots, n,
\end{gathered}
$$

where $D_{i k}, h_{i k m}$ and $C_{i}$ represents inertia, Coriolis/centrifugal and gravity terms (Vundavilli et al 2007).

Once the torque required at different joints are calculated, the amount of power consumed at those joints can be determined. At a given joint $i$, the required mechanical power is calculated as $P_{i}=\frac{1}{T} \int_{0}^{T}\left|\tau_{i} \dot{q}_{i}\right| d t$, where $T$ is the cycle time. Therefore, the total power consumed can be determined as follows:

$$
P=\sum_{i=1}^{n} P_{i}
$$

where $n$ is the number of joints. In the above expression, the power consumption during the walking of the robot has been considered, which includes inertia, gravitational, Coriolis and

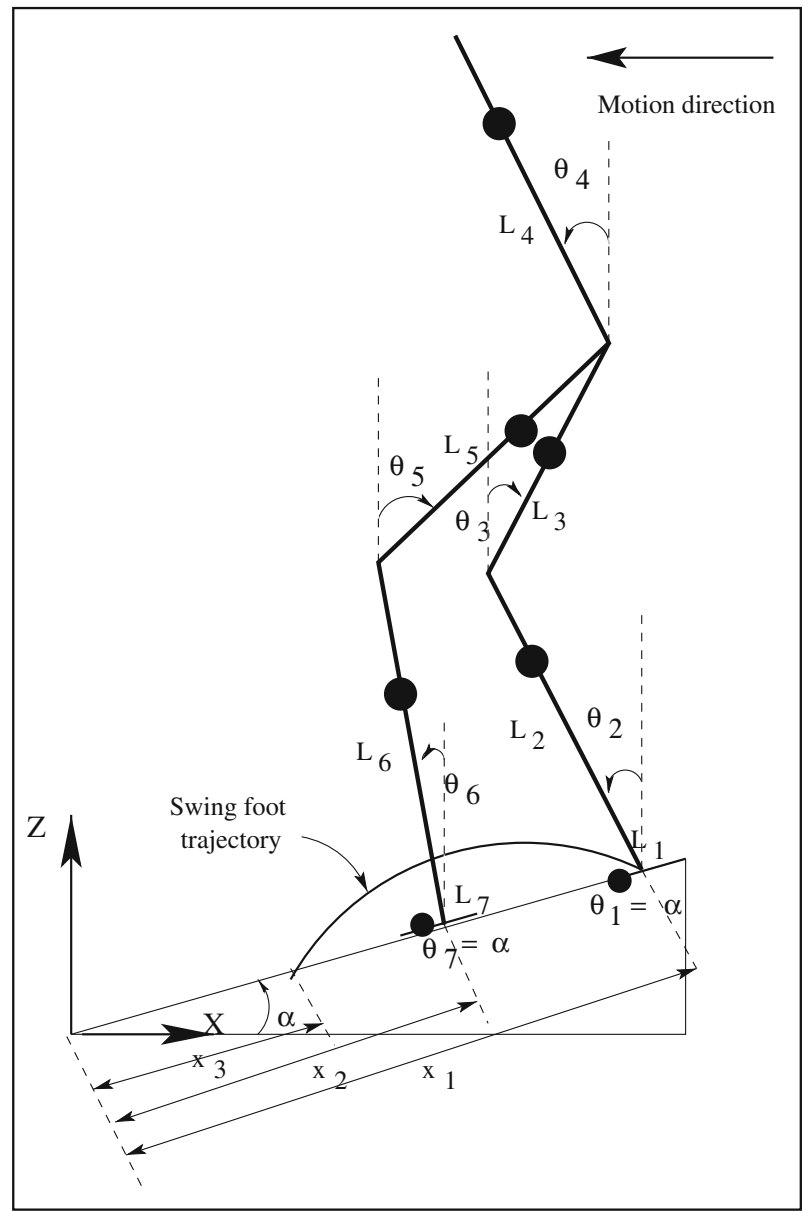

Figure 4. A schematic view of a two-legged robot(7-DOF) moving down the sloping surface. 
centrifugal effects. However, the said power term does not consider the dissipation of power for static postures to simplify the model.

\subsection{Descending the sloping surface}

The method of gait generation for descending the sloping surface is kept similar to that of the ascending case. However, there are some variations in the formulation, as discussed below. The schematic view of the biped robot descending the sloping surface is shown in figure 4 . The same cubic polynomial trajectory for the swing leg is assumed. The only difference lies in the constraints or boundary conditions, which are given as follows:

- at $x=x_{1} \cos \alpha, z=x_{1} \sin \alpha$,

- at $x=\left(x_{1} \cos \alpha+x_{2} \cos \alpha\right) / 2, z=x_{1} \sin \alpha+\frac{f_{s}}{2}$,

- at $x=\left(x_{2} \cos \alpha+x_{3} \cos \alpha\right) / 2, z=x_{2} \sin \alpha+\frac{f_{s}}{2}$,

- at $x=x_{3} \cos \alpha, z=x_{3} \sin \alpha$.

The hip trajectory, lower link's gait and trunk motion are generated in the similar way as have been done for the ascending case. It is important to note that there is a small change in the expression of ZMP. It is so, because of the fact that the acceleration due to gravity $g$ and $\ddot{z}_{i}$ are acting in the same direction. The expression of ZMP for descending the sloping surface can be written as follows:

$$
x_{Z M P}=\frac{\sum_{i=1}^{7}\left(I_{i} \dot{\omega}_{i}+m_{i} x_{i}\left(\ddot{z}_{i}+g\right)-m_{i} \ddot{x}_{i} z_{i}\right)}{\sum_{i=1}^{7} m_{i}\left(\ddot{z}_{i}+g\right)},
$$

where the terms indicate their usual meaning.

The method of torque calculation remains the same, but the D-H parameter angles are to be modified according to the new posture and these are found to be as follows: $q_{1}=\theta_{1}=\alpha$ (as there is no variation), $q_{2}=\left(-90+\theta_{2}+\theta_{1}\right), q_{3}=\left(\theta_{3}-\theta_{2}\right), q_{4}=\left(\theta_{4}-\theta_{3}\right), q_{5}=\left(\theta_{5}-\theta_{4}\right), q_{6}=$ $\left(\theta_{6}-\theta_{5}\right), q_{7}=\left(\theta_{7}-\left(90+\theta_{6}\right)\right)$. The expressions for inertia and Coriolis/centrifugal terms will be the same with those for ascending the sloping surface. The only difference is with the gravity terms, as the masses are moving in the direction of gravity (Vundavilli et al 2007).

\section{Results and discussion}

The validity of the above mathematical model for ascending and descending through the sloping surface is tested with the help of a case study.

Table 1. Parameters for different links of the biped robot.

\begin{tabular}{lcccc}
\hline Link & $\mathrm{m}(\mathrm{kg})$ & $\mathrm{L}(\mathrm{m})$ & $\mathrm{r}(\mathrm{m})$ & $\mathrm{I}\left(\mathrm{kg} \mathrm{m^{2 }}\right)$ \\
\hline 1 & 0.5 & 0.06 & 0.02000 & 0.000600 \\
2 & 2.0 & 0.34 & 0.20000 & 0.021067 \\
3 & 5.0 & 0.30 & 0.24000 & 0.078000 \\
4 & 30.0 & 0.60 & 0.48000 & 1.872000 \\
5 & 5.0 & 0.30 & 0.24000 & 0.078000 \\
6 & 2.0 & 0.34 & 0.20000 & 0.021067 \\
7 & 0.5 & 0.06 & 0.02000 & 0.000600 \\
\hline
\end{tabular}




\subsection{Case study}

The parameters related to different links of the biped robot considered in this work are given in table 1 .
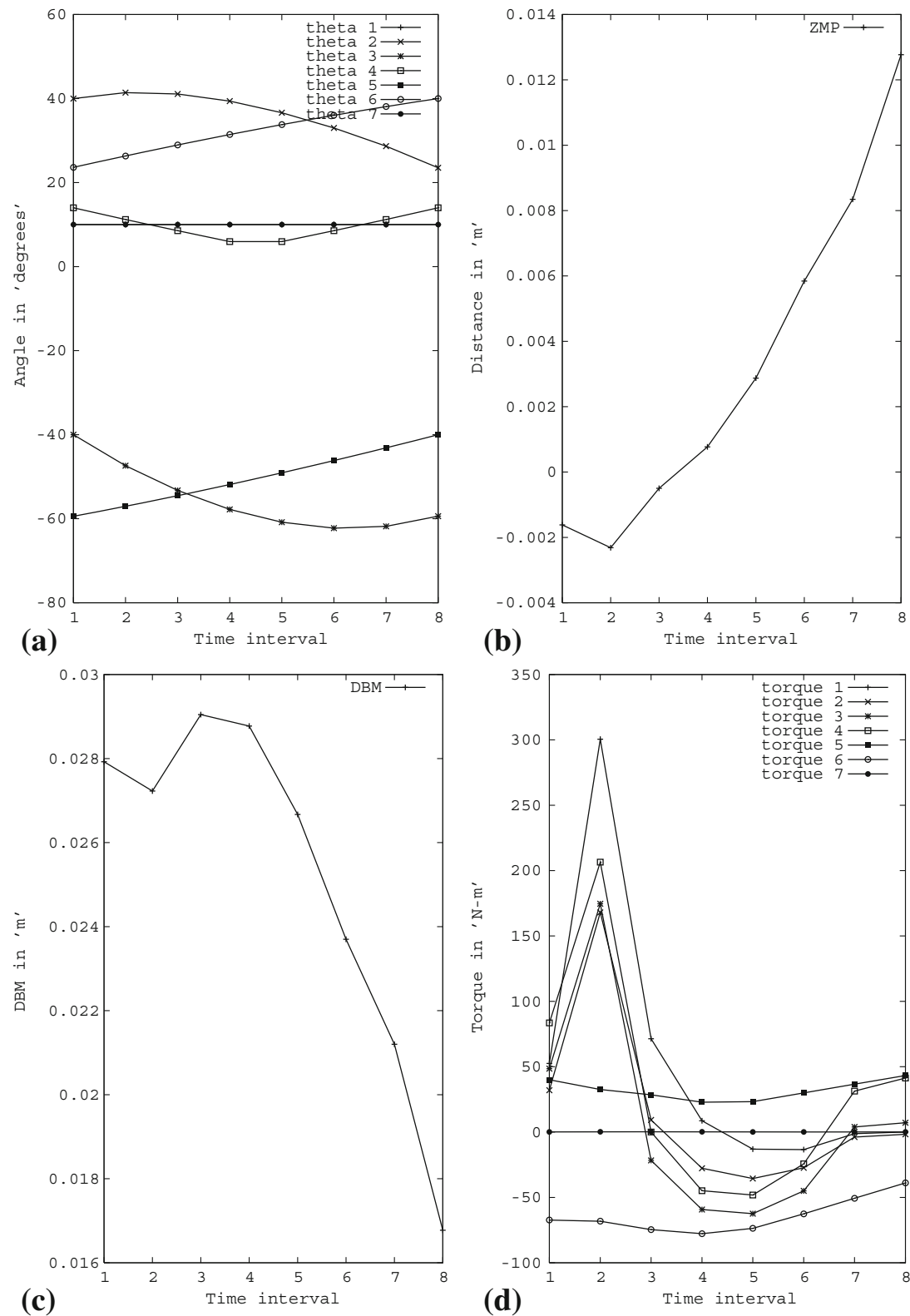

Figure 5. Variations of joint angles, ZMP, DBM and joint torques, while ascending the sloping surface in one complete cycle. (a) Variation of joint angles. (b) Variation of ZMP. (c) Variation of DBM. (d) Variation of joint torques. 
3.1a Ascending the sloping surface: The inputs fed to the algorithm are the foot placements (that is, $x_{1}, x_{2}, x_{3}$ ) of the biped robot along the sloping surface measured with respect to the global coordinate system fixed at the starting point of the slope and initial posture of the robot
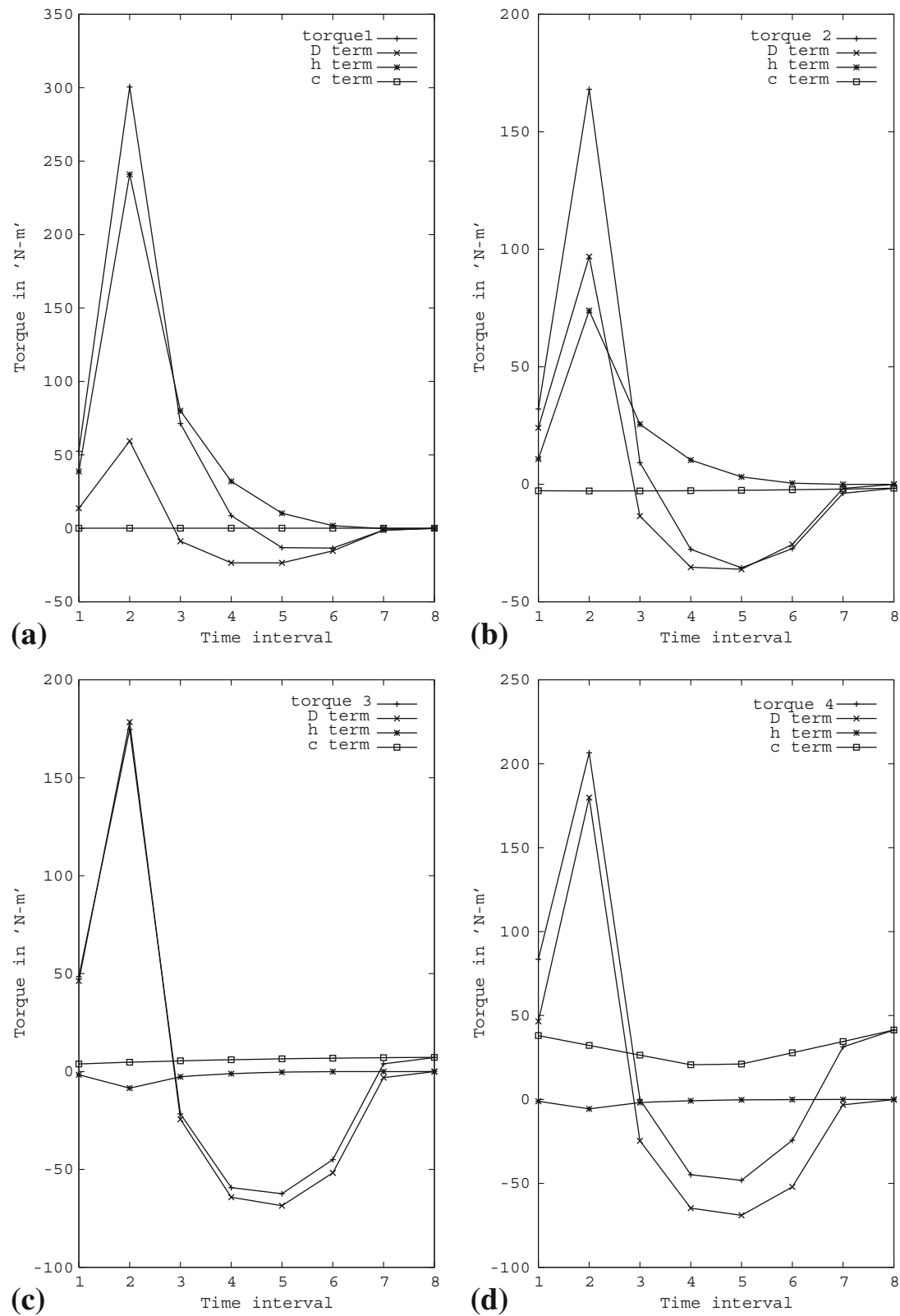

Figure 6. Contributions of $\mathrm{D}, \mathrm{h}$ and $\mathrm{C}$ on different joint torques during ascending a sloping surface. (a) Contribution of $\mathrm{D}, \mathrm{h}$ and $\mathrm{C}$ on torque 1. (b) Contribution of $\mathrm{D}, \mathrm{h}$ and $\mathrm{C}$ on torque 2. (c) Contribution of D, $\mathrm{h}$ and $\mathrm{C}$ on torque 3. (d) Contribution of $\mathrm{D}, \mathrm{h}$ and $\mathrm{C}$ on torque 4 . (e) Contribution of $\mathrm{D}, \mathrm{h}$ and $\mathrm{C}$ on torque 5. (f) Contribution of $\mathrm{D}, \mathrm{h}$ and $\mathrm{C}$ on torque 6. (g) Contribution of $\mathrm{D}, \mathrm{h}$ and $\mathrm{C}$ on torque 7. 
(that is, $\theta_{2}$ and $\theta_{3}$ of the swing leg). Let us assume that the inputs are as follows: $x_{1}=0.03 \mathrm{~m}$, $x_{2}=0.18 \mathrm{~m}, x_{3}=0.33 \mathrm{~m}$ and $\theta_{2}=40^{\circ}$ and $\theta_{3}=-40^{\circ}$.

The maximum angle of the slope along which the biped robot can walk without any slip can be determined using the value of static friction coefficient, say $\mu=0.4$. Thus, the maximum

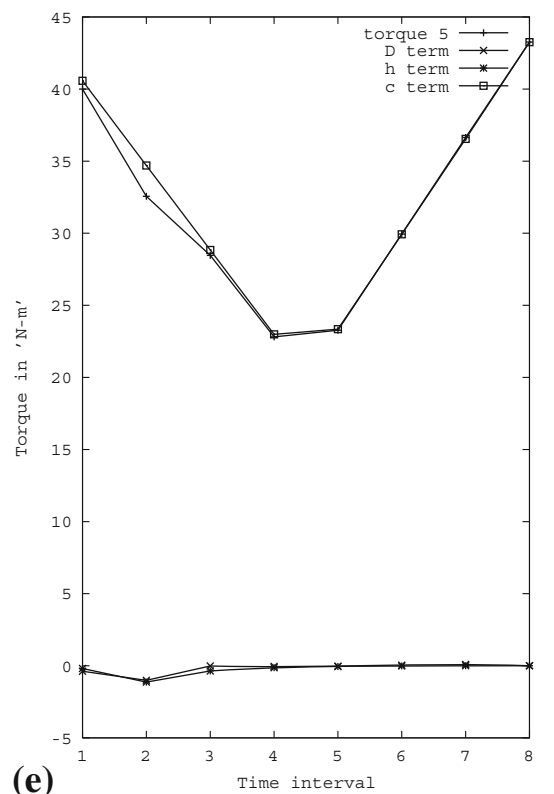

(e)
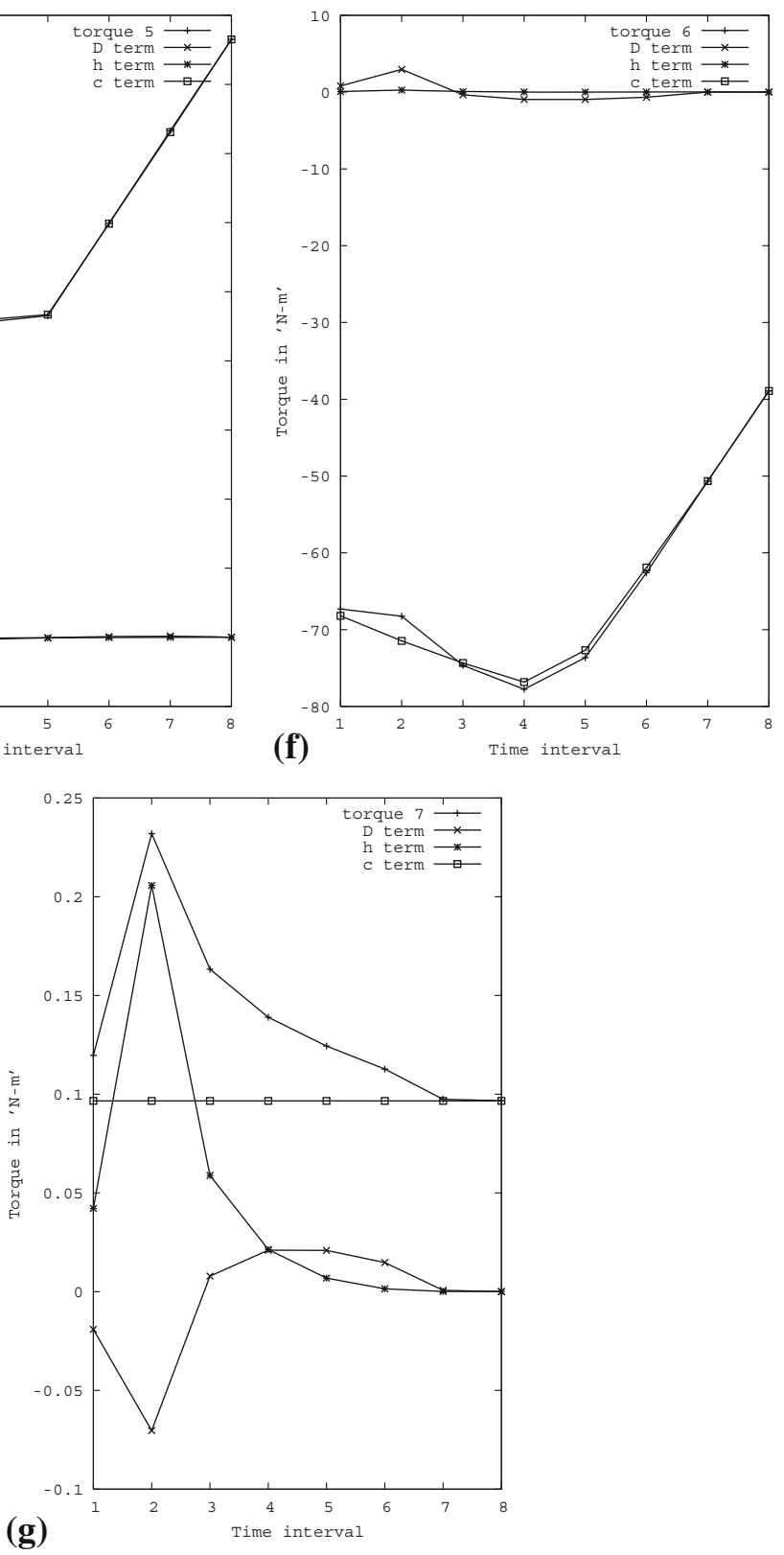

Figure 6. (continued). 
angle of slope along which the robot can walk without slip is found to be equal to $\tan ^{-1}(\mu)$ $=\tan ^{-1}(0.4)=21.8^{\circ}$. In the present study, the slope of the surface has been kept fixed to $10^{\circ}$.

Figures 5 through 14 show the results of computer simulations. The variations of joint angles during ascending the sloping surface are shown in figure 5 (a). As the angles of the swing foot
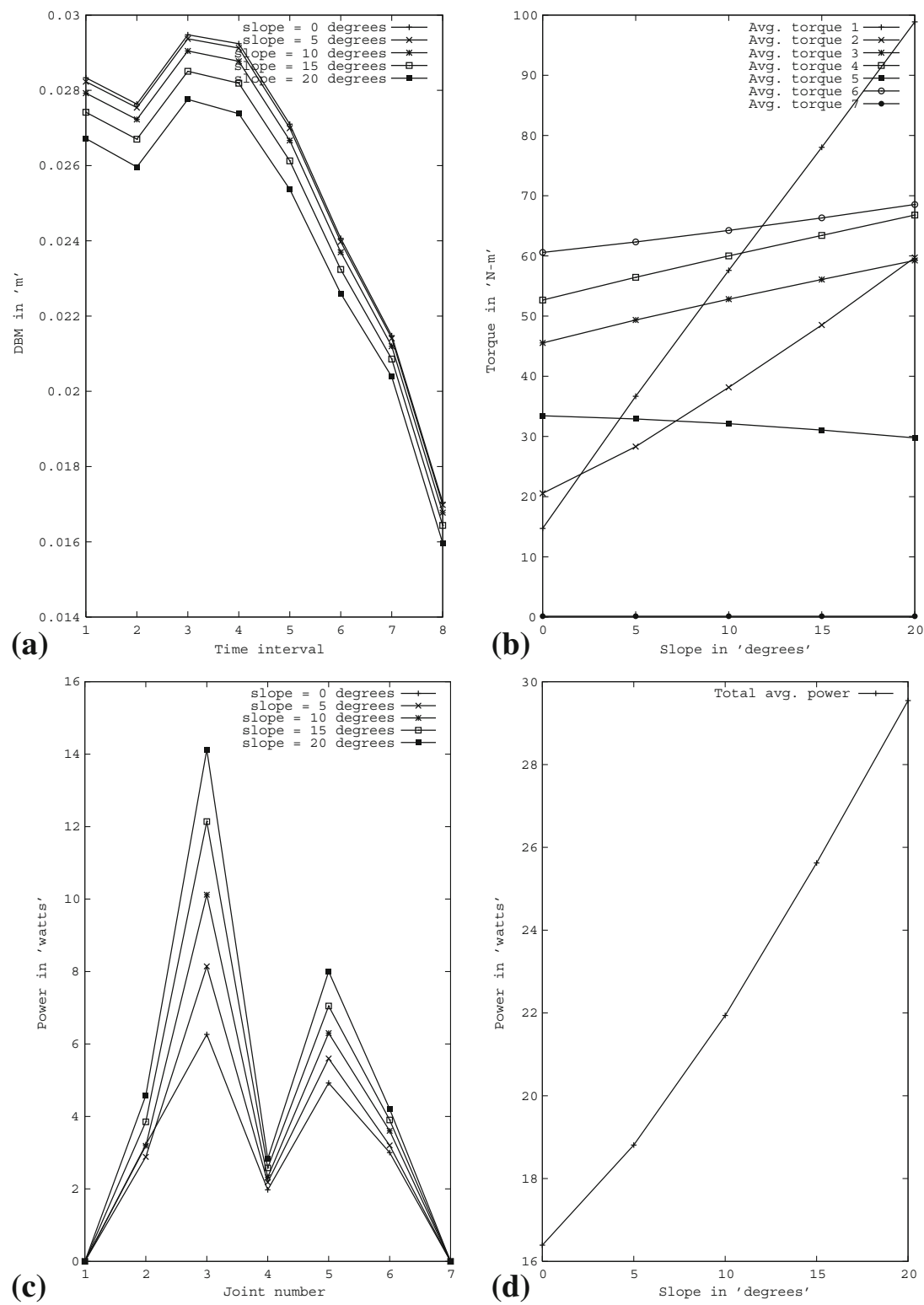

Figure 7. Effect of variation of slope angle on DBM, average torques, average power consumption and total power consumption during ascending the sloping surface. (a) Variation of DBM. (b) Variation of average torques at different joints. (c) Variations of average power at different joints. (d) Variation of total power. 
and supporting foot (that is, $\theta_{1}$ and $\theta_{7}$ ) are kept fixed (no variation) and assumed to be equal to the slope of the surface, two lines indicating the variations of $\theta_{1}$ and $\theta_{7}$ are overlapping at $10^{\circ}$, as shown in the above mentioned figure. The rest of the joints follow the repeatability conditions to obtain the cyclic gait. The variations of ZMP and DBM in one complete cycle are shown in
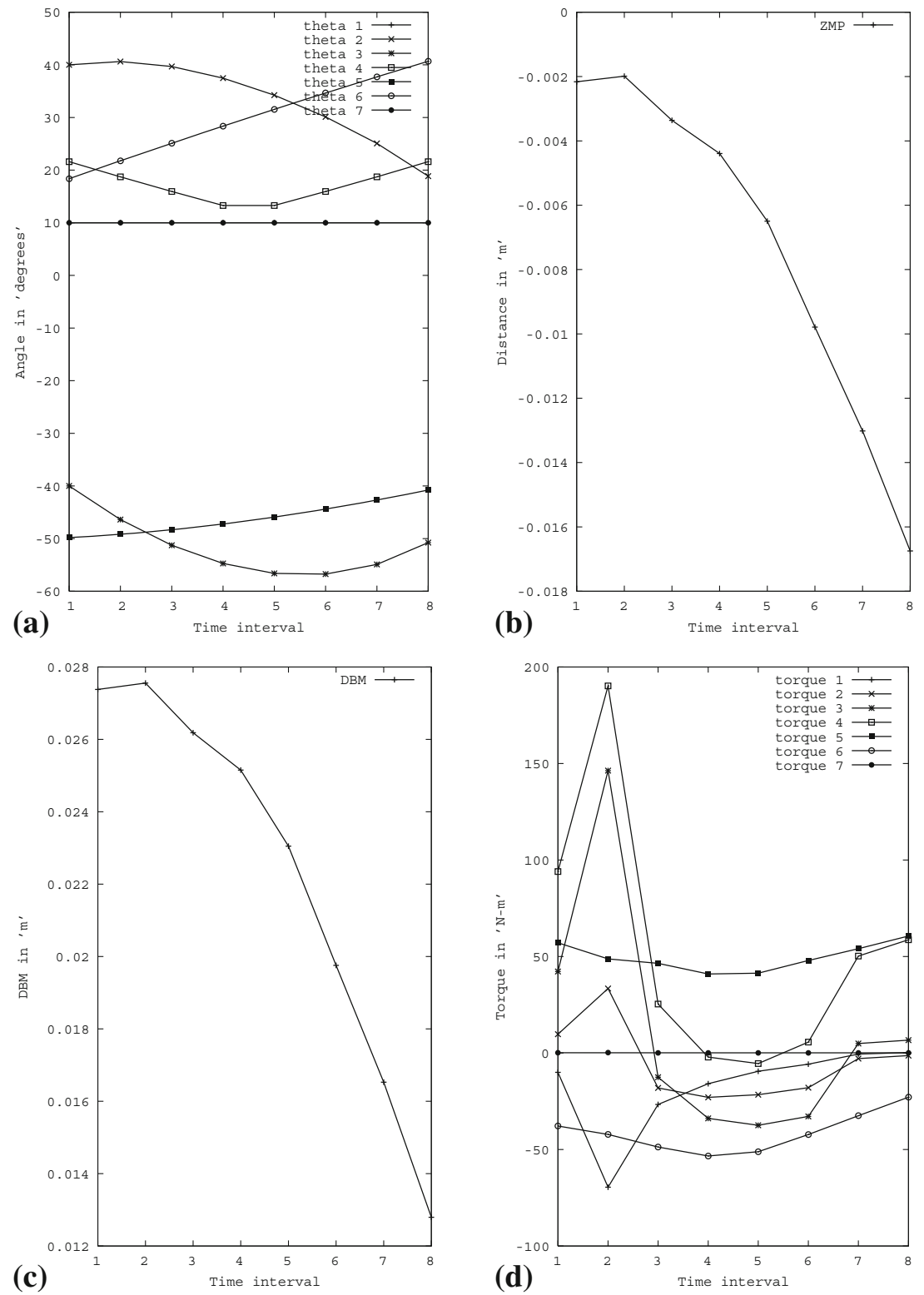

Figure 8. Variations of joint angles, ZMP, DBM and joint torques, while descending the sloping surface in one complete cycle. (a) Variation of joint angles. (b) Variation of ZMP. (c) Variation of DBM. (d) Variation of joint torques. 
figures 5(b) and 5(c), respectively. The $x_{Z M P}$ is initially found to lie at the back of the ankle joint. It can be observed that during the second time interval, the $x_{Z M P}$ is further moving towards the back from the ankle joint. This may happen due to the fact that the hip joint is still behind the
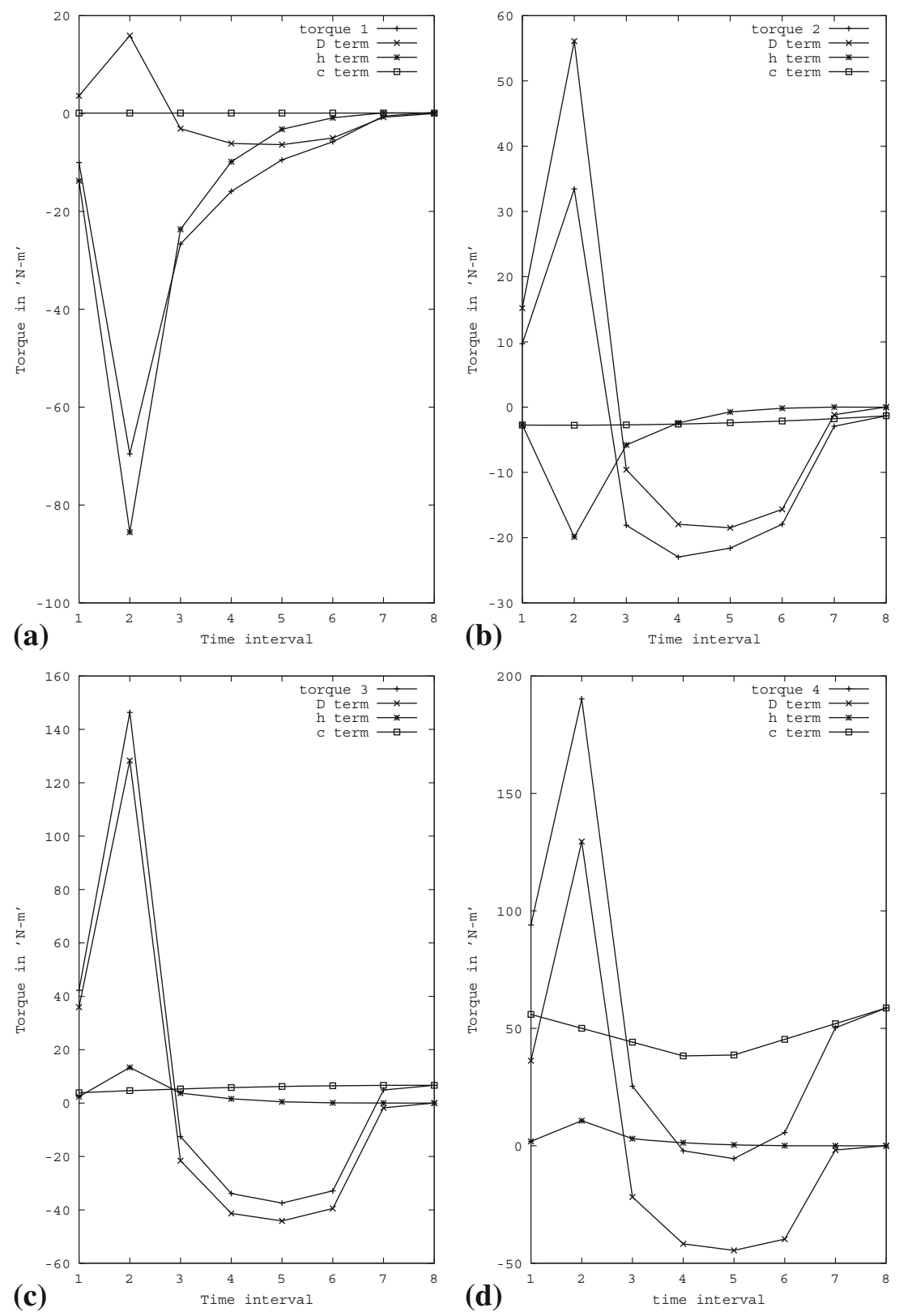

Figure 9. Contributions of $\mathrm{D}, \mathrm{h}$ and $\mathrm{C}$ on different joint torques during descending a sloping surface. (a) Contribution of D, h and C on torque 1. (b) Contribution of D, h and C on torque 2. (c) Contribution of D, $\mathrm{h}$ and $\mathrm{C}$ on torque 3. (d) Contribution of $\mathrm{D}, \mathrm{h}$ and $\mathrm{C}$ on torque 4. (e) Contribution of $\mathrm{D}, \mathrm{h}$ and $\mathrm{C}$ on torque 5. (f) Contribution of D, h and C on torque 6. (g) Contribution of D, h and C on torque 7. 
supporting foot. Due to this effect, the DBM value during the second time interval is showing a sudden fall. From this point onwards, the $x_{Z M P}$ is moving towards the ankle joint and gradually towards the end of the foot sole in the direction of motion, as the swing leg reaches to the next step. It happens due to the moments generated by the moving masses.
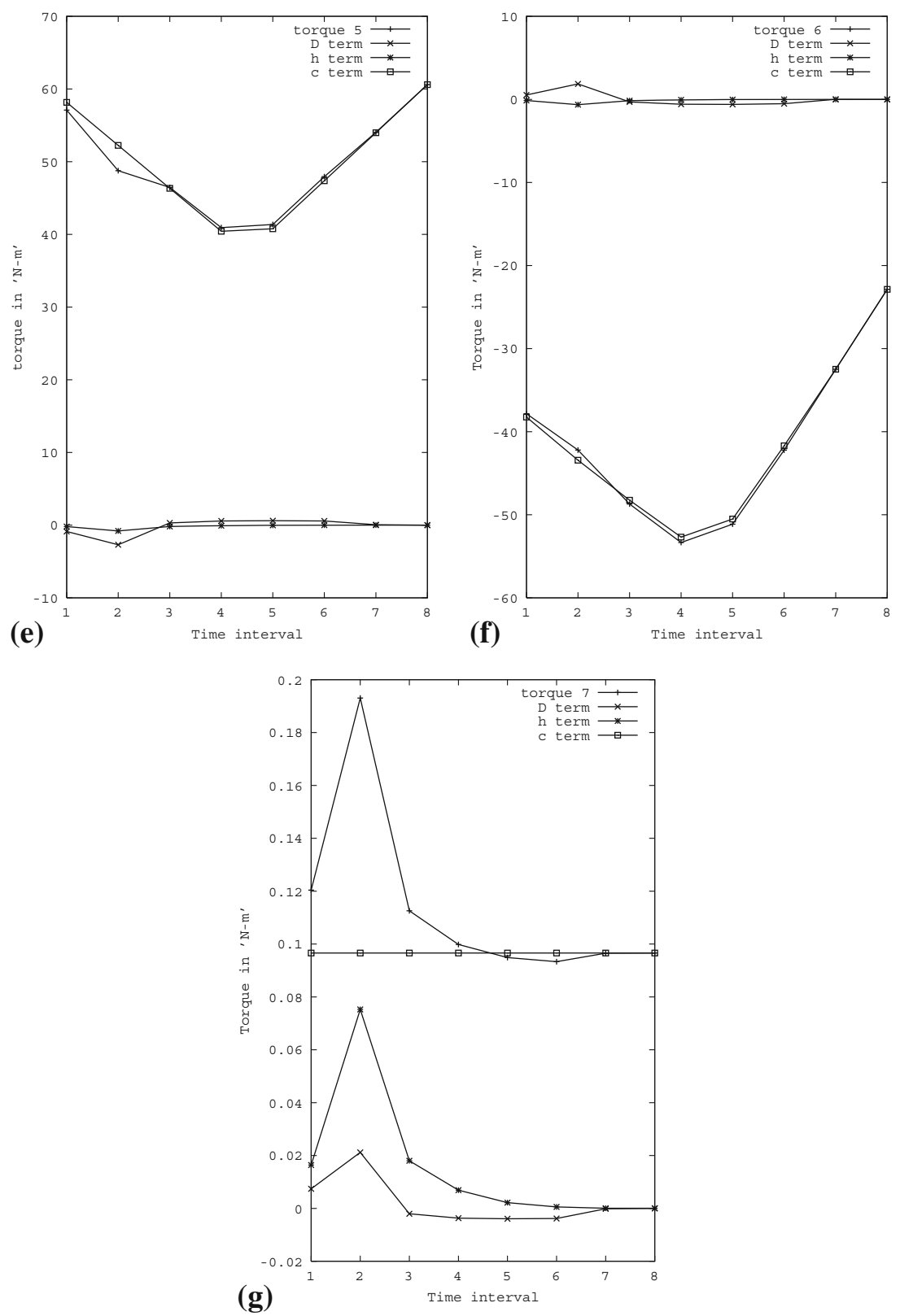

Figure 9. (continued). 
It is observed that for the entire motion cycle, the ZMP point is always lying inside the foot support polygon. Therefore, the generated gait is said to be dynamically balanced. The DBM value is found to be increasing initially and then decreasing towards the end of the cycle (refer
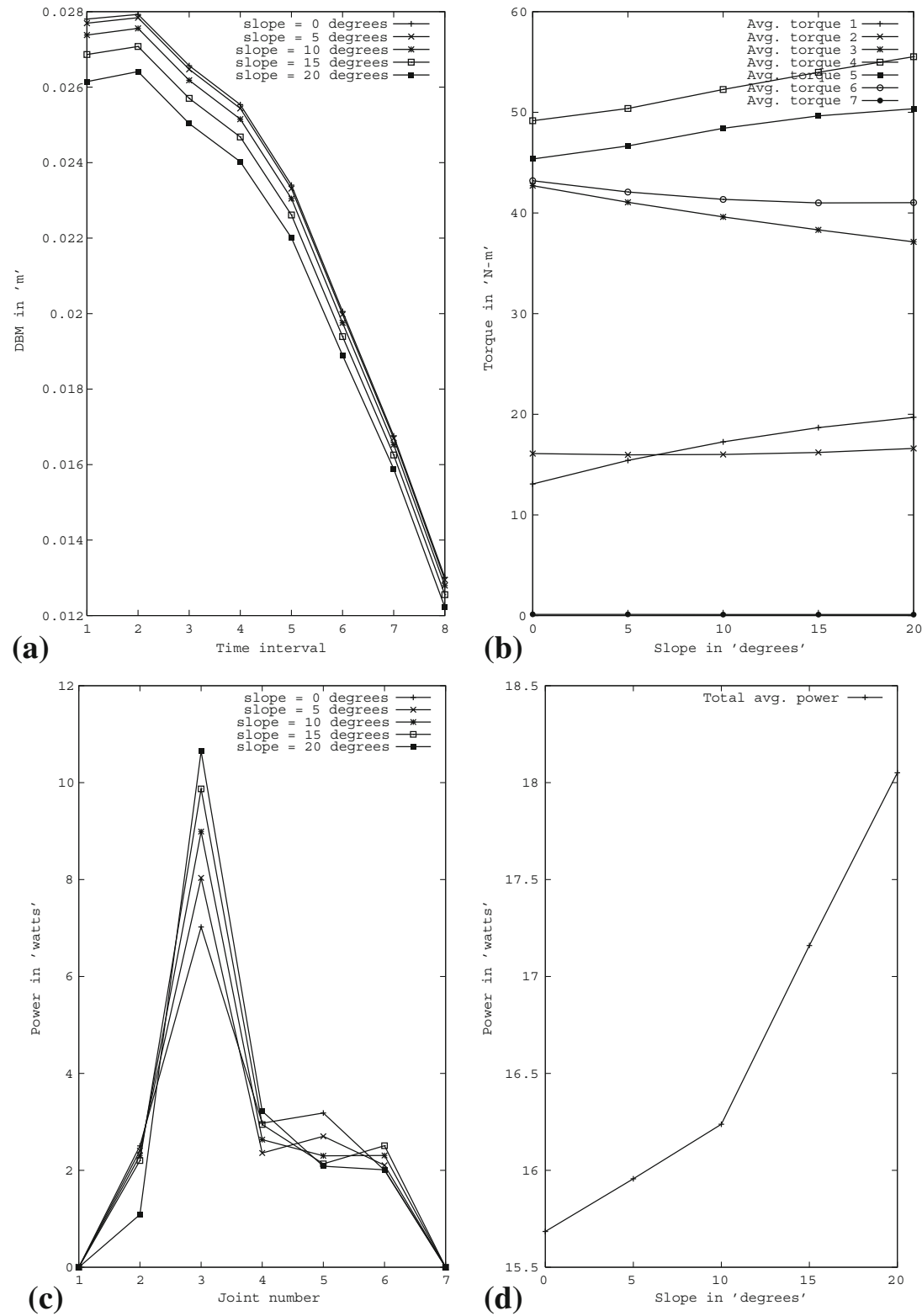

Figure 10. Effect of variation of slope angle on DBM, average torques, average power consumption and total power consumption during descending the sloping surface. (a) Variation of DBM. (b) Variation of average torques at different joints. (c) Variations of average power at different joints. (d) Variation of total power. 


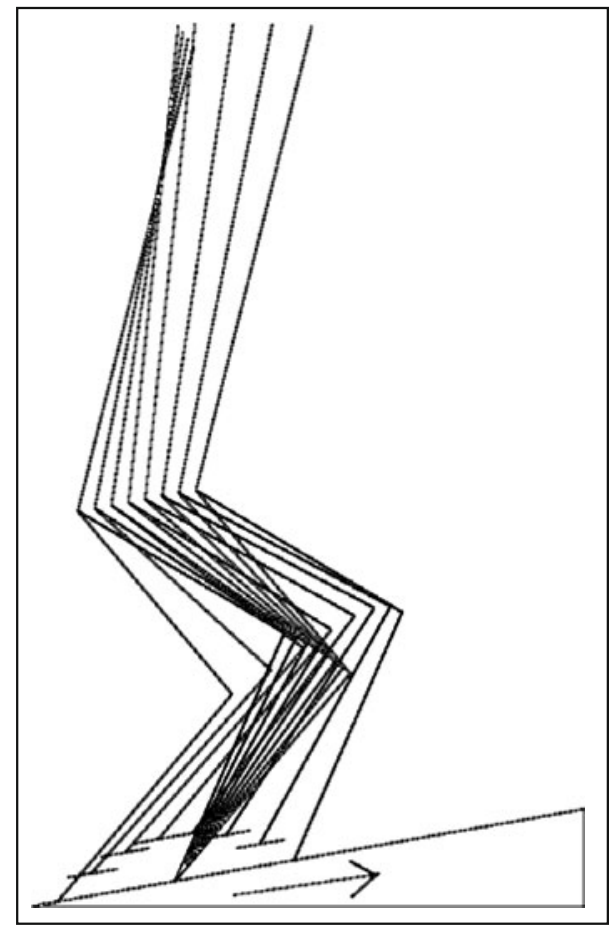

Figure 11. Simulation related to ascending the sloping surface in one cycle.

to figure 5 (c)). This happens due to the fact that the $x_{Z M P}$ points are moving gradually towards the end of the sole through the ankle joint.

The variation of torques at different joints are shown in figure 5(d). It is to be noted that clockwise torque is considered as the positive and anti-clockwise torque is assumed to be the negative. It is also interesting to note that the torque values of ankle, knee and hip joints of the swing leg and trunk during the second time interval are coming out to be relatively more, compared to their values at other time intervals. It happens due to the fact that the inertia and centrifugal/Coriolis effects are found to be more at this interval, as the biped robot starts moving from the initial posture. The contribution of inertia, Coriolis/centrifugal and gravity terms on different joint torques are shown in figure 6. It is interesting to note that the effect of inertia and centrifugal/Coriolis components are more and that of gravity is negligible at the starting of the swing phase on $\tau_{2}$ and $\tau_{3}$. It is also interesting to notice that both the gravity as well as inertia have considerable effect on $\tau_{4}$. Moreover, it is observed that the effect of gravity is more on $\tau_{5}$ and $\tau_{6}$ but the effects of centrifugal and inertia terms are found to be negligible.

The variations of DBM, average torques, average power consumption at different joints and total power consumption with the slope of the surface are shown in figure 7. It is observed from figure 7(a) that the DBM values are reducing with the increase of slope of the surface. It may be due to the fact that the projected area of the foot supporting region is reducing, as the slope increases. Figure 7(b) shows the variation of average torque required at different joints with the angle of slope. It is to be noted that the average torques required at all the joints are increasing 


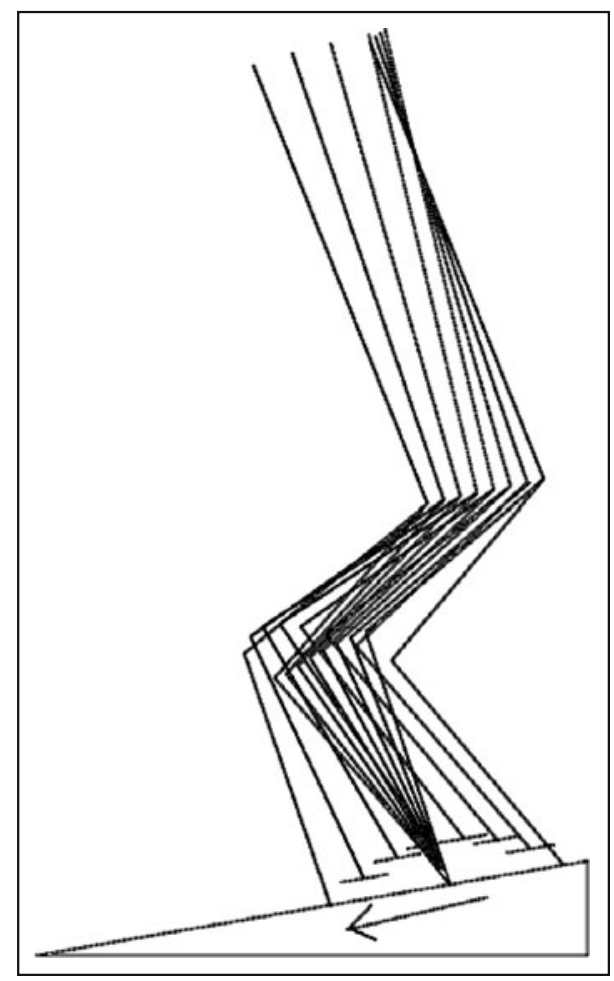

Figure 12. Simulation related to descending the sloping surface in one cycle.

with the slope of the surface except $\tau_{5}$. It may be due to the reason that the included angle between the upper part of the supporting leg and the trunk reduces, as the slope of the surface is increasing. The variations of average power at different joints and total power consumption during ascending the sloping surface are shown in figures 7(c) and (d), respectively. It is to be observed that the power consumption increases with the increase in slope of the surface, as expected.

3.1b Descending the sloping surface: Let us consider the following inputs: $x_{1}=0.33 \mathrm{~m}, x_{2}=$ $0.18 \mathrm{~m}, x_{3}=0.03 \mathrm{~m}$ and $\theta_{2}=40^{\circ}$ and $\theta_{3}=-40^{\circ}$. The slope of the surface has also been assumed to be equal to $10^{\circ}$. The variations of the joint angles required for descending the sloping surface are shown in figure 8(a). As both $\theta_{1}$ and $\theta_{7}$ are assumed to be equal to the angle of slope (that is, no variation) during a complete cycle, they are overlapping on each other. Figure 8(b) shows the variation of $x_{Z M P}$ in one cycle. It is to be noticed that the location of the $x_{Z M P}$ is always lying on the front side of the ankle joint and moving towards the end of the supporting foot except at the second interval. It may be due to the reason that the projection of hip joint is lying behind the supporting foot. As the $x_{Z M P}$ is moving towards the end of the foot, the DBM values are reducing towards the end of the cycle, as shown in figure 8(c).

The variations of different joint torques are displayed in figure $8(\mathrm{~d})$. It is interesting to observe that the torque values at the second time interval for the ankle, knee and hip joints of the swing 


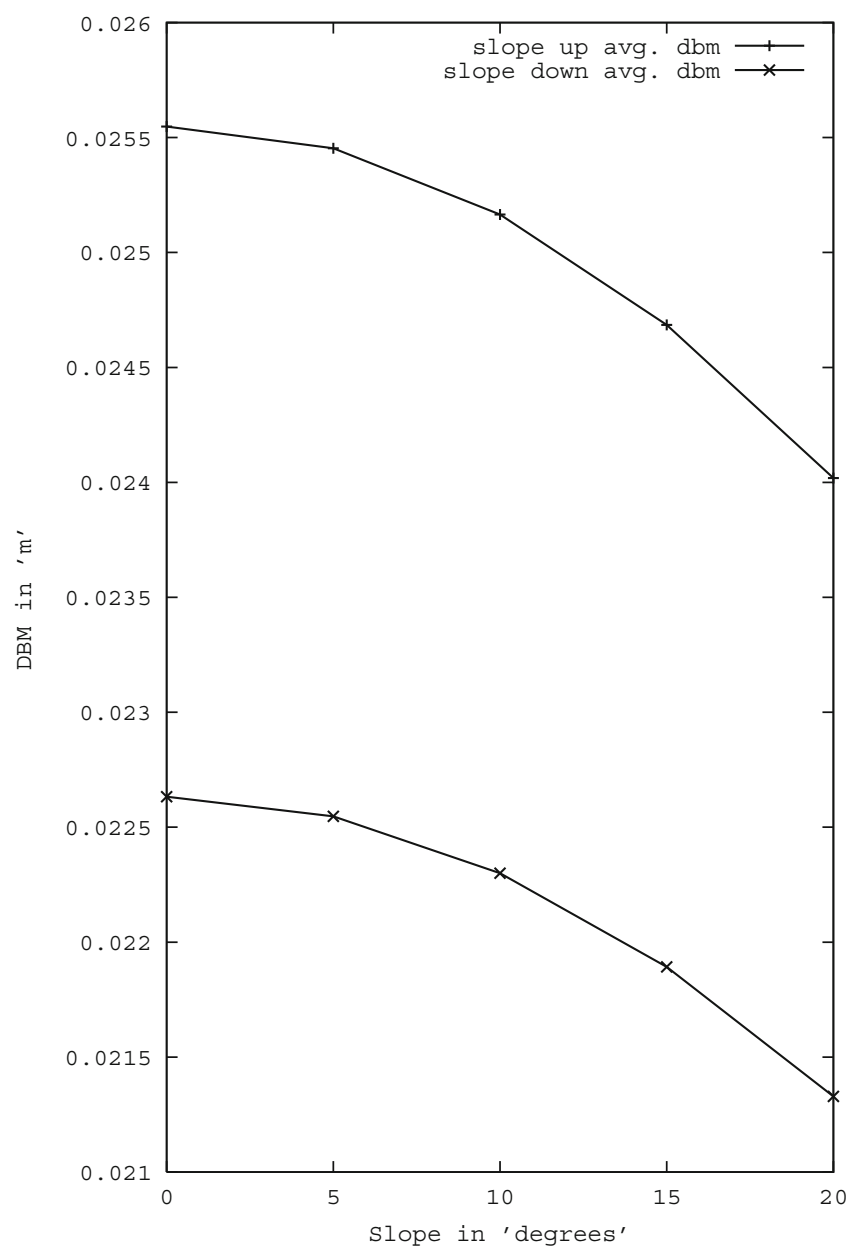

Figure 13. Variations of average DBM with the angle of slope for both the ascending and descending cases.

leg and trunk are more compared to the torques at other intervals. It may be due to the fact that the inertia and centrifugal/Coriolis components are having significant effect during that interval, as the biped robot starts swinging its leg from the initial posture. The effect of inertia, centrifugal/Coriolis and gravity components on different joint torques are shown in figure 9. It is interesting to note that the inertia and centrifugal/Coriolis components have significant contributions on the joint torques of the swing leg, whereas the joint torques of the ground leg are mainly influenced by the inertia and gravity terms. However, the contributions of different components on the joint torques in case of ascending the sloping surface are seen to be slightly different from those observed in case of descending, as expected.

The effect of variations of slope of the surface on DBM, average torque, average power and total power are displayed in figure 10. The DBM values at different intervals are seen to be reduced, as the slope of the surface increases (refer to figure 10(a)). This happens due to the reason that the area of projected ground foot polygon is reducing, as the slope of the surface 


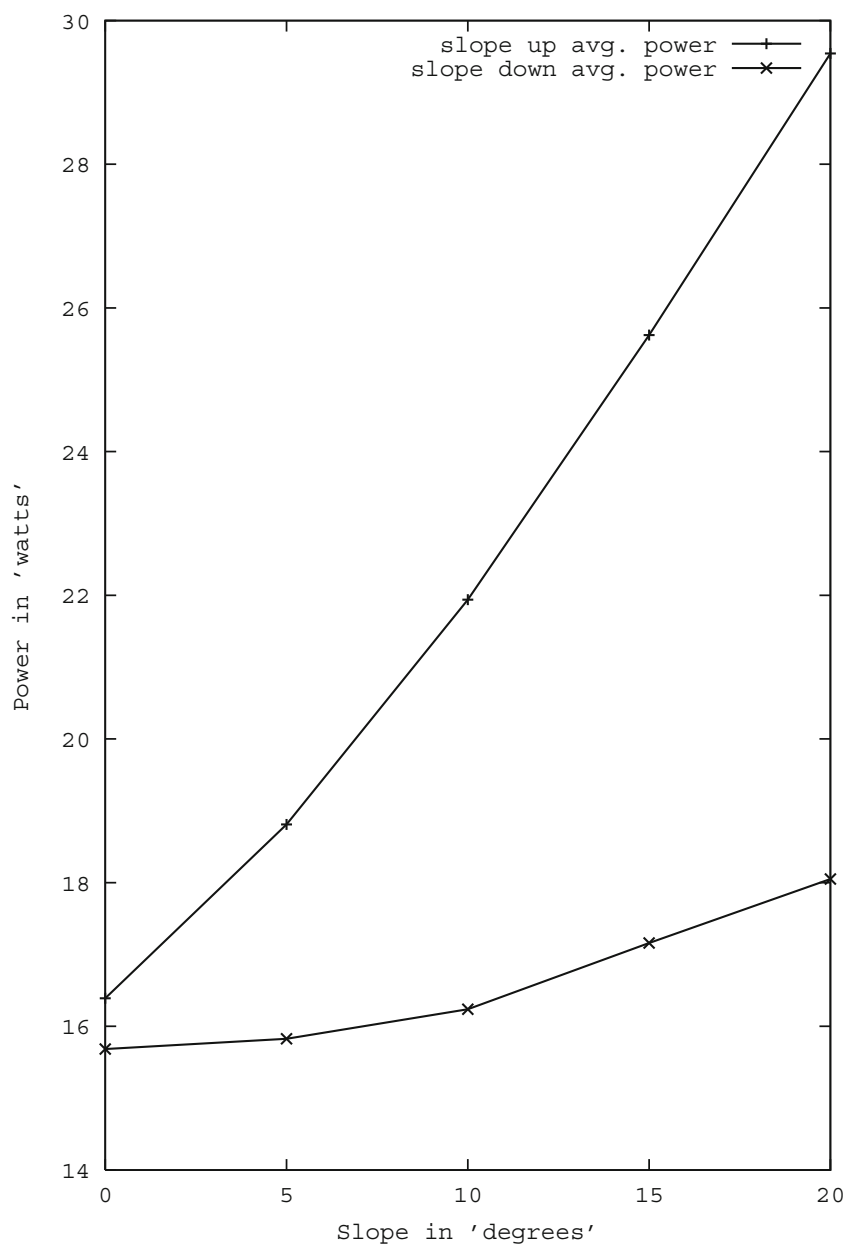

Figure 14. Variations of power consumption with the angle of slope for both the ascending and descending cases.

increases. Figure 10(b) shows the variations of average torque at different joints with the increase of slope. The average torque required at different joints (except for $\tau_{3}$ and $\tau_{6}$ ) are found to increase with the angle of slope. This may be due to the reason that the angle variation at these two joints are decreasing, as it is moving down the slope. Figures 10(c) and (d) show the variations of average power at different joints and total power required with the increase of the angle of slope, respectively. The average power and total power required for descending are found to increase with the angle of slope. It is interesting to note that the rate of increase in total power requirement with the angle of slope comes out to be more in ascending case compared to that in descending case.

3.1c Comparison: The results of the simulation related to ascending and descending the sloping surface are shown in figures 11 and 12 . It is interesting to observe that the repeatability 
conditions for gait generation are achieved. Figure 13 shows the variations of average DBM with the angle of slope for both the ascending and descending cases. The average DBM values for the descending case are always found to be less than those of the ascending case. It may be concluded that the latter is more balanced than the former, which matches exactly with our general experience in moving through the sloping surface.

The variations of power consumption with the angle of slope have been compared for the ascending and descending cases (refer to figure 14). The power consumption in ascending case is found to be more than that of the descending case, as expected. It happens due to the fact that the robot moves against the gravity in the former case.

\section{Concluding remarks}

From the above study, the following conclusions have been made:

- Simulation results show that the generated gait is cyclic in nature.

- The generated gait is seen to be dynamically balanced, as the ZMP is found to lie within the projected area of foot support polygon.

- It has been observed that the ascending gait is dynamically more balanced and consumes more power compared to the descending gait does. The above observations match exactly with our general experience of moving through the sloping surface.

- It has also been observed that the robot becomes dynamically less stable and consumes more power with the increase of the angle of slope of the surface, in both the ascending as well as descending cases.

It is to be noted that the present study has a significant value for real robot motion synthesis. The obtained ranges of joint angle changes and joint torques have the meaning for the choice of the motors and evaluation of their required motion speed (combining the walking speed with the ranges of joint displacements).

\section{Scope for future work}

The present work may be extended in a number of ways, as indicated below.

- The performance of the developed model has been tested through computer simulations only. It will be more interesting to carry out experiments with the real robot. However, attention has to be paid on the following issues to make the experimentation possible with the real robot: online modelling and realization of the environment with the help of a camera and image analysis; force or inertia sensors mounted on the foot to determine the angle of slope; texture analysis to determine the static friction coefficient of the surface; design and development of closed-loop control system; modelling of foot-ground reaction as a multi-DOF system; and others.

- A 7-DOF biped robot has been considered in the present work. However, it may be extended for a biped robot having more than 7-DOF to implement the movement of the robot in the lateral plane.

- An attempt can also be made to solve the dynamically balanced gait generation problems of a biped robot on-line, on the sloping surface. The authors are working on the above issues, at present. 


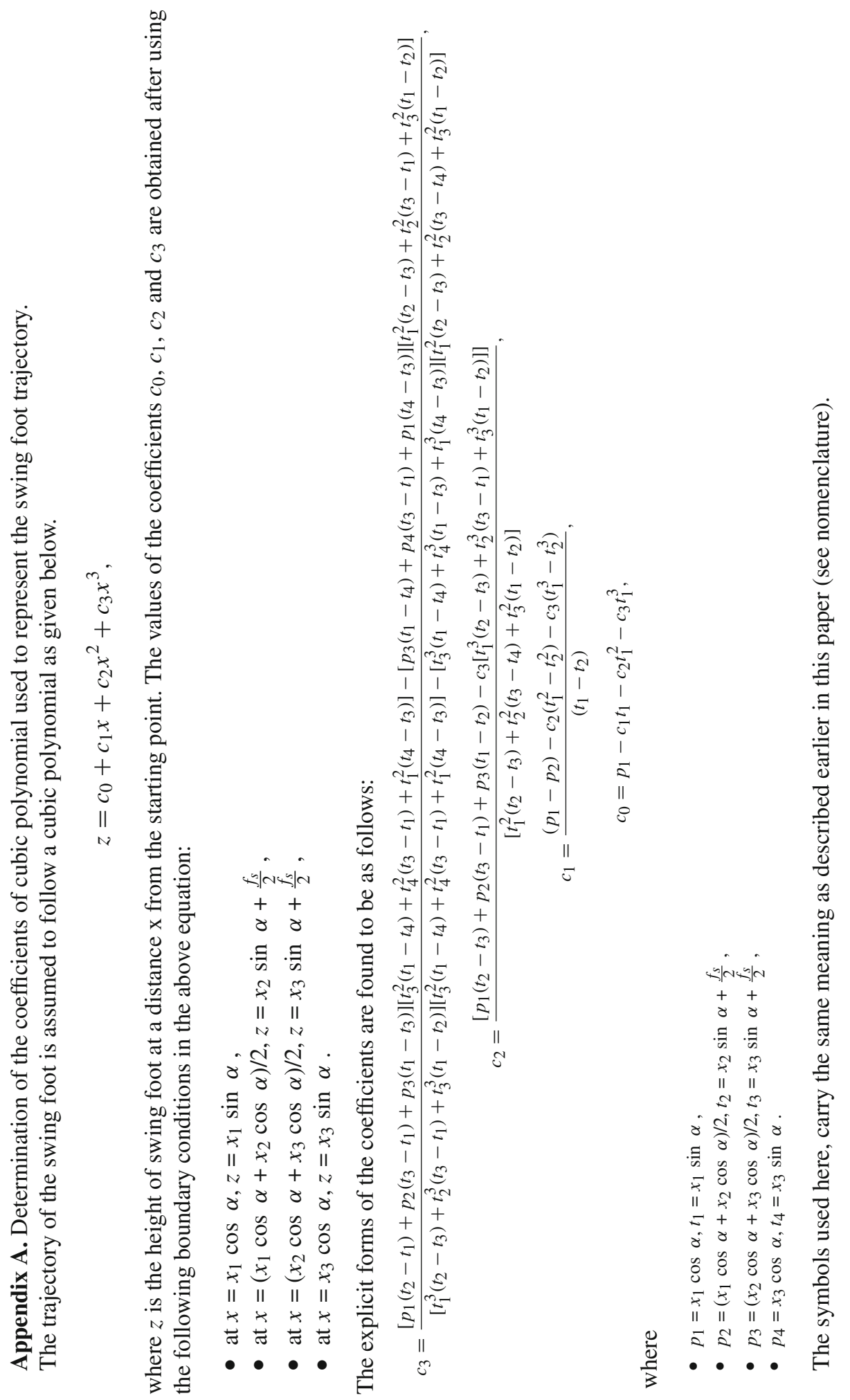




\section{Nomenclature}

\begin{tabular}{|c|c|}
\hline$C$ & Gravity terms in torque equation \\
\hline$D$ & Inertia terms in torque equation \\
\hline$F$ & Ground reaction force, $\mathrm{N}$ \\
\hline$F_{N}$ & Normal reaction force, $\mathrm{N}$ \\
\hline$F_{T}$ & Friction force, $\mathrm{N}$ \\
\hline$f_{s}$ & Length of the foot, $\mathrm{m}$ \\
\hline$g$ & Acceleration due to gravity, $\mathrm{m} / \mathrm{s}^{2}$ \\
\hline$h$ & Coriolis and centrifugal terms in torque equation \\
\hline$h_{1}, h_{2}$ & Hip heights, m \\
\hline$I_{1}, \ldots, I_{7}$ & Moment of inertia of the links, $\mathrm{kg}-\mathrm{m}^{2}$ \\
\hline$l_{1}, l_{2}$ & Distance between the ankle joint and the projection of hip joint, $\mathrm{m}$ \\
\hline$L_{1}, \ldots, L_{7}$ & Link lengths, $\mathrm{m}$ \\
\hline$m_{1}, \ldots, m_{7}$ & Lumped masses of links, $\mathrm{kg}$ \\
\hline$p$ & Projection of mass center, $\mathrm{m}$ \\
\hline$q_{1}, \ldots, q_{7}$ & Included angle between the links in D-H parameter setting, degrees \\
\hline$r_{1}, \ldots, r_{7}$ & Distance of lumped masses from the joints, $\mathrm{m}$ \\
\hline$t$ & Time step, s \\
\hline$T$ & Total time of travel, s \\
\hline$V_{\max }$ & Maximum velocity of the swing leg, $\mathrm{m} / \mathrm{s}$ \\
\hline$x_{D B M}$ & Dynamic balance margin in the direction of motion, $\mathrm{m}$ \\
\hline $\begin{array}{l}x_{Z M P} \\
\text { Greek letters }\end{array}$ & Distance of zero moment point from the ankle joint in $\mathrm{x}$-direction, $\mathrm{m}$ \\
\hline$\alpha$ & Angle of slope, degrees \\
\hline$\mu$ & Coefficient of friction \\
\hline$\phi$ & Friction angle, degrees \\
\hline$\tau$ & Torque, N-m \\
\hline$\theta$ & Joint angle, degrees \\
\hline \multicolumn{2}{|c|}{ Abbreviations } \\
\hline$C G$ & Center of Gravity \\
\hline$D O F$ & Degrees of Freedom \\
\hline$D B M$ & Dynamic Balance Margin \\
\hline FRI & Foot Rotation Indicator \\
\hline$Z M P$ & Zero-Moment Point \\
\hline
\end{tabular}

\section{References}

Boone, G N, Hodgins, J K 1997 Slipping and tripping reflexes for bipedal robots, Autonomous Robots, 4: 259-271

Fu, K S, Gonzalez, R C, Lee, C S G 1987 ROBOTICS Control, Sensing, Vision, and Intelligence, McGrawHill International Edition, Industrial Engineering series

Furusho, J, Masubuchi, M 1987 A theoretically motivated reduced order model for the control of Dynamic biped locomotion, Transactions of the ASME J. of Dynamic Systems, Measurement and Control, 109: $155-163$

Goswami, A 1999 Foot rotation indicator (FRI) Point: A new gait planning tool to evaluate postural stability of biped robots, in Proc. of IEEE Int. Conf. on Robotics and Automation, Detroit, Michigan, pp. 47-52 
Juricic, D, Vukobratovic, M 1972 Mathematical modeling of biped walking systems, ASME Publication, 72-WA/BHF13

Kajita, S, Kanehiro, F, Kaneko, K, Fujiwara, K, Harada, K, Yokoi, K, Hirukawa, H 2003 Biped walking pattern generation by using preview control of zero-moment point, in Proc. of IEEE Int. Conf. on Robotics and Automation, Taipei, Taiwan, Sept 14-19, pp. 1620-1626

Kajita, S, Tani, K 1991 Study of dynamic biped locomotion on rugged terrain- derivation and application of the linear inverted pendulum mode, in Proc. of IEEE Int. Conf. on Robotics and Automation, Sacramento, California, pp. 1405-1411

Kim, J Y, Park, I W, lee, J, Kim, M S, Cho, B K, Oh, J H 2005 System design and dynamically walking of humanoid robot KHR-2, in Proc. of IEEE Int. Conf. on Robotics and Automation, Barcelone, Spain, pp. 1443-1448

Kim, J Y, Park, I W, Oh, J H 2007 Walking control algorithm of biped humanoid robot on uneven and inclined floor, J. of Int. Robot Syst., 48: 457-484

Lim, H, Kaneshima, Y, Takanishi, A 2002 On line walking pattern generation for biped humanoid robot with trunk, in Proc. of IEEE Int. Conf. on Robotics and Automation, Washington, DC, pp. 3111-3116

Lum, H K, Zribi, M, Soh, Y C 1999 Planning and control of a biped robot", Int. J. of Engineering Sciences, 37: 1319-1349

Ono, T, Murakami, T, Ohnishi, K 1998 An approach to biped robot control according to surface condition of ground, IEEE Int. workshop on Advanced Motion Control, Coimbra, Jun 29-Jul 1, pp. 129-134

Pleaten, F, Jessy, W G, Westervelt, R, Gabrial, A 2003 Stable walking of a 7-DOF biped robot, IEEE Transactions on Robotics and Automation, 19(4): 653-668

Pratt, J, Chew, C M, Torres, A, Dilworth, P, Pratt, G 2001 Virtual model control: An intuitive approach for bipedal locomotion, Int. J. of Robotics Research, 20(2): 129-143

Seo, Y J, Yoon, Y S 1995 Design of a robust dynamic gait of the biped using the concept of dynamic stability margin", Robotica, 13, Cambridge University press, pp. 461-468

Silva, F M, Tenreiro Machado, J A 1998 Towards efficient biped robots, in Proc. of IEEE/RSJ Int. Conf. on Intelligent Robots and Systems, Victoria, B.C., Canada, pp. 394-399

Sugahara, Y, Mikuriya, Y, Hashimoto, K, Hosobata, T, Sunazuka, H, Kawase, M 2005 Walking control method of biped locomotors on inclined plane, in Proc. of IEEE Int. Conf. on Robotics and Automation, Barcelona, Spain, pp. 1977-1982

Sugihara, T, Nakamura, Y, Inoue, H 2002 Real time humanoid motion generation through ZMP manipulation based on inverted pendulum control, in Proc. of IEEE Int. Conf. on Robotics and Automation, Washington, DC, pp. 1404-1409

Takanishi, A, Tochizawa, M, Karaki, H, Kato, I 1989 Dynamic biped walking stabilized with optimal trunk and waist motion, IEEE/RSJ International Workshop on Intelligent Robots and Systems'89, Tsukuba, Japan, Sept. 4-6, pp. 187-192

Vukobratovic, M, Borovac, B, Surla, D, Stokic, D 1990 Biped locomotion, dynamics, stability, control and applications, Springer-Verlag, Berlin

Vukobratovic, M, Frank, A A, Juricic, D 1970 On the stability of biped locomotion, IEEE Trans. Biomedical Engineering, BME 17(1): 25-36

Vukobratovic, M, Radic, A D 2004 Contribution to the integrated control of artificial human gait, SISY-2004, Serbia and Montenegro, Oct 1-2

Vundavilli, P R, Sahu, S K, Pratihar, D K 2007 Dynamically balanced ascending and descending gaits of a two-legged robot, Int. J. of Humanoid Robotics, 4(4): 717-751

Zheng, Y F, Shen, J 1990 Gait synthesis for the SD-2 biped robot to climb slopping surface, IEEE Transactions on Robotics and Automation, 6(1): 86-96

Zheng, Y F, Shen, J, Sias, F R 1998 A motion control scheme for a biped robot to climb sloping surfaces, in Proc. of IEEE Int. Conf. on Robotics and Automation, pp. 814-816

Zhou, C, Yue, P K, Ni, J, Chan, S B 2004 Dynamically stable gait planning for a humanoid robot to climb sloping surface, in Proc. of IEEE Conf. on Robotics, Automation and Mechatronics, Singapore, Dec 1-3, pp. 341-346 